\title{
PLANES, BRANES AND AUTOMORPHISMS II. BRANES IN MOTION
}

\author{
BS ACHARYA, JM FIGUEROA-O'FARRILL, B SPENCE, AND S STANCIU
}

\begin{abstract}
We complete the classification of supersymmetric configurations of two M5-branes, started by Ohta and Townsend. The novel configurations not considered before are those in which the two branes are moving relative to one another. These configurations are obtained by starting with two coincident branes and Lorentz-transforming one of them while preserving some supersymmetry. We completely classify the supersymmetric configurations involving two M5-branes, and interpret them group-theoretically. We also present some partial results on supersymmetric configurations involving an arbitrary number of M5-branes. We show that these configurations correspond to Cayley planes in eightdimensions which are null-rotated relative to each other in the remaining $(2+1)$ dimensions. The generic configuration preserves $\frac{1}{32}$ of the supersymmetry, but other fractions (up to $\frac{1}{4}$ ) are possible by restricting the planes to certain subsets of the Cayley grassmannian. We discuss some examples with fractions $\frac{1}{32}, \frac{1}{16}, \frac{3}{32}, \frac{1}{8}$, and $\frac{1}{4}$ involving an arbitrary number of branes, as well as their associated geometries.
\end{abstract}

\section{Contents}

1. Introduction 2

2. The statement of the problem 3

3. Supersymmetric intersections of two M5-branes 4

3.1. A convenient normal form for Lorentz transformations 5

3.2. Conditions for supersymmetry 6

3.3. Supersymmetry and null rotations 8

3.4. Group-theoretical analysis 11

4. Null rotations and an exotic spinor isotropy group 12

4.1. An explicit real realisation of $\mathrm{C}_{1,10} \quad 12$

4.2. Spinor isotropies 14

4.3. An exotic spinor isotropy group 14

$\begin{array}{ll}\text { 4.4. Invariants } & 15\end{array}$

4.5. More on vectors and spinors 17

$\begin{array}{ll}\text { 5. Multiple intersections } & 17\end{array}$

5.1. G-relatedness 18

5.2. An equivalent eight-dimensional problem 19

Date: June 19, 2021. 
5.3. The group-theoretical fraction $\nu_{K} \quad 20$

5.4. Some examples and their geometries 21

6. Summary and Outlook 23

Acknowledgements $\quad 25$

References $\quad 25$

\section{INTRODUCTION}

This is Part II in a series of papers dedicated to the group-theoretical study of intersecting brane configurations. In the first paper in this series, hereafter referred to as Part I [1], we outlined a complete characterisation of configurations of multiply intersecting static branes in terms of subgroups of $\operatorname{Spin}_{10}$ preserving some spinors. In the present paper we will consider configurations in which the branes are not necessarily static relative to each other. We refer the reader to Part I and to the references therein for a summary of the literature on this topic as well as for the basic notation.

This paper is organised as follows. In Section 2 we will state the problem to be addressed, namely the determination of all supersymmetric configurations of $m$ intersecting M5-branes in eleven-dimensional Minkowski spacetime, as well as of the fraction $\nu$ of the supersymmetry which is preserved. In Section 3 we solve this problem for the special case of $m=2$ branes, and as preparation for the following sections, we interpret the solutions group-theoretically. This relies heavily on the results of Part I and on those in [9]. We find new supersymmetric configurations corresponding to supersymmetric branes at angles which have then been null-rotated relative to each other. These new configurations trace their origin to the fact that there exists a class of singular orbits in the spinorial representation of $\operatorname{Spin}_{10,1}$ whose isotropy does not leave invariant any time-like directions. In Section 4 we study this spinorial representation in some detail, and collect some basic facts concerning this exotic isotropy group. In Section 5 we tackle the multiple intersection problem. We start by reformulating the problem of multiple intersections of moving branes in eight-dimensional terms. We will show that all such supersymmetric configurations consist of Cayley planes in eight dimensions which have been null-rotated in the remaining three dimensions. These configurations generically preserve $\frac{1}{32}$ of the supersymmetry; but by restricting the planes to sub-orbits of the Cayley grassmannian, it is possible to find configurations which preserve a larger fraction - the largest such fraction being $\frac{1}{4}$. In Section 6 we discuss some examples of such configurations and explore their geometries. Section 7 concludes this paper with a summary of the status of the classification problem. 


\section{The STATEMENT OF THE PROBLEM}

In this section we set up the problem. Let us consider the M5brane solution for definiteness. Let $\left(x^{\mu}\right)$ denote the eleven-dimensional coordinates, where $\left(x^{0}, x^{1}, \ldots, x^{5}\right)$ are coordinates along the brane and $\left(x^{6}, \ldots, x^{9}, x^{\natural}\right)$ are coordinates transverse to the brane. Far away from the brane, the metric is asymptotically flat, so that the Killing spinors of the supergravity solution have constant asymptotic values $\varepsilon$, obeying

$$
\Gamma_{012345} \varepsilon=\varepsilon,
$$

where $\varepsilon$ is a real 32-component spinor of $\operatorname{Spin}_{10,1}$. We think of $\operatorname{Spin}_{10,1}$ as contained in the Clifford algebra $C \ell_{1,10}$ generated by the $\Gamma_{M}$. Then the spinor representation $\Delta$ of $\operatorname{Spin}_{10,1}$ can and will be identified once and for all with one of the two inequivalent irreducible representations of $\mathrm{C}_{1,10}$.

As in Part I we will rewrite (1) in a more convenient notation. Let us then fix a point $x$ in the spacetime $M$ and an orthonormal frame $e_{0}, e_{1}, \ldots, e_{9}, e_{\natural}$ for the tangent space at $x$. This allows us to identify the tangent space $T_{x} M$ with eleven-dimensional Minkowski spacetime $M^{10,1}$. The tangent space to the worldvolume of a fivebrane passing through $x$ will be a $(5,1)$-dimensional oriented (and time-oriented) subspace of $T_{x} M$, or equivalently an oriented (and timeoriented) $(5,1)$-plane in $M^{10,1}$. The space of such planes is the grassmannian $\mathrm{SO}_{10,1}^{0} / \mathrm{SO}_{5,1}^{0} \times \mathrm{SO}_{5}$, where $\mathrm{SO}^{0}$ stands for the connected component of the identity. If $v_{0}, v_{1}, \ldots, v_{5}$ is an orthonormal basis for such a plane, we can construct a $(5,1)$-vector $\pi=v_{0} \wedge v_{1} \wedge \cdots \wedge v_{5}$ in $\wedge^{5,1} M^{10,1}$ which has unit norm. Conversely, to any given unit simple $(5,1)$-vector $\pi=v_{0} \wedge v_{1} \wedge \cdots \wedge v_{5}$, we associate an oriented time-oriented (5, 1)-plane given by the span of the $v_{i}$. The condition for supersymmetry (1) can be rewritten more generally as

$$
\pi \cdot \varepsilon=\varepsilon,
$$

where $\cdot$ stands for Clifford multiplication and where we have used implicitly the isomorphism of the Clifford algebra $\mathrm{C} \ell_{1,10}$ with the exterior algebra $\bigwedge M^{10,1}$. When $\pi=e_{0} \wedge e_{1} \wedge \cdots \wedge e_{5}$, equation (2) agrees with equation (1).

Because $\pi$ has unit norm, $\pi \cdot \pi=\mathbb{1}$, and because it has zero trace, the subspace $\Delta(\pi) \subset \Delta$ defined by

$$
\Delta(\pi) \equiv\{\varepsilon \in \Delta \mid \pi \cdot \varepsilon=\varepsilon\}
$$

is sixteen-dimensional. In other words, the M5-brane preserves one half of the supersymmetry. Now let $\eta$ be another $(5,1)$-plane. It also preserves one half of the supersymmetry, but both planes taken together will in general preserve a smaller fraction $\nu$ defined to be the number of solutions to both (2) and the analogous equation for $\eta$. In other words, 
$32 \nu$ is the dimension of the subspace

$$
\Delta(\pi \cup \eta) \equiv \Delta(\pi) \cap \Delta(\eta) \subset \Delta .
$$

Clearly we are not restricted to only two branes. Indeed, consider $m$ branes with tangent planes $\pi_{1} \equiv \pi, \pi_{2}, \ldots, \pi_{m}$. We say that the configuration $\cup_{i=1}^{m} \pi_{i}$ is supersymmetric if and only if

$$
\Delta\left(\cup_{i=1}^{m} \pi_{i}\right)=\bigcap_{i=1}^{m} \Delta\left(\pi_{i}\right) \neq\{0\} ;
$$

the fraction $\nu$ of the supersymmetry which is preserved being determined according to

$$
32 \nu=\operatorname{dim} \Delta\left(\cup_{i=1}^{m} \pi_{i}\right) .
$$

A priori $\nu$ can only take the values $\frac{1}{32}, \frac{1}{16}, \frac{3}{32}, \ldots, \frac{1}{2}$; although only the following fractions are known to occur: $\frac{1}{32}, \frac{1}{16}, \frac{3}{32}, \frac{1}{8}, \frac{5}{32}, \frac{3}{16}, \frac{1}{4}$ and $\frac{1}{2}$. The two fundamental questions are the following.

Question 1. How can one characterise the supersymmetric configurations $\cup_{i=1}^{m} \pi_{i}$ ?

Question 2. What fraction $\nu$ of the supersymmetry is preserved by a given supersymmetric configuration $\cup_{i=1}^{m} \pi_{i}$ ?

For the special case of branes which are not moving relative to each other, so that $\pi_{i}=e_{0} \wedge \xi_{i}$, where $\xi_{i}$ are 5-planes in $e_{0}^{\perp} \cong \mathbb{E}^{10}$, then both questions have been answered fully for $m=2$ in [9] (see also [1]). In [2] (see also [6]) we answered the first question for all $m$, using techniques of calibrated geometry. In Part I we gave a partial answer to the second question for arbitrary $m$, recasting the problem in terms of group theory. In the present paper we will lift the condition that the branes be static and consider arbitrary configurations of two or more branes. We will answer both questions fully for $m=2$, and will present some partial results for general $m$.

\section{Supersymmetric intersections of Two M5-BRAneS}

In this section we analyse the conditions for supersymmetry of a configuration of two intersecting M5-branes. We consider a starting configuration in which both branes are parallel, with tangent plane $\pi$. This configuration is supersymmetric, preserving one half of the supersymmetry. Now suppose that we perform a Lorentz transformation to one of the branes. Then the two fundamental questions are subsumed into one.

Question 3. For which Lorentz transformations $L \in \mathrm{SO}_{10,1}^{0}$ will the configuration with tangents $\pi$ and $L \pi$ be supersymmetric and what fraction $\nu$ of the supersymmetry will be preserved? 
In order to answer this question it is first convenient to put $L$ in a standard form which can be easily parametrised. In the simpler case when $L$ is a rotation in $\mathrm{SO}_{10}$, a normal form is given by letting $L$ lie in a fixed maximal torus. The conjugacy theorem for maximal tori guarantee that this is always possible after a change of basis. Since $\mathrm{SO}_{10,1}^{0}$ is noncompact it will not have a maximal torus, but we can still do something similar.

\subsection{A convenient normal form for Lorentz transformations.}

Suppose we are given two branes related by a Lorentz transformation $L$ in $\mathrm{SO}_{10,1}^{0}$. We can undo this transformation in the following way: we first transform one of the branes so that it is no longer moving relative to the other one, and then we simply realign them with a rotation. Working backwards now, we can reach the configuration $L \pi$ from $\pi$ by first rotating $\pi \mapsto R \pi$ and then boosting. ${ }^{1}$ However because of the Lorentz invariance of the worldvolume of the branes themselves, a boost will only change the configuration if it is in a direction normal to both branes. In other words, if we were to perform a boost, say, in a direction contained in $\pi \cup R \pi$, then this boost can be undone by a further rotation in that subspace and by a boost along one of the branes. Hence the relative configuration between the branes will not change. This is not to say that the configurations are physically indistinguishable, since when two branes intersect, Lorentz invariance on the brane is broken and one can detect one brane moving relative to the other brane. However the relative configuration of their worldvolumes does not change and neither will its supersymmetry. In practice, what this means is that if $\pi=e_{0} \wedge \xi$ and $R \pi=e_{0} \wedge R \xi$, then for a boost to effect any change in the configuration, it has to be along a vector in $\xi^{\perp} \cap R \xi^{\perp}$. Therefore for a generic rotation $R$ in $\mathrm{SO}_{10}$, so that $\xi^{\perp} \cap R \xi^{\perp}=\{0\}$, no such boost would be possible. In other words, in order to effect any change in the relative configuration of the branes by a boost, it will be necessary for the initial rotation to belong to $\mathrm{SO}_{9}$.

We are always free to choose a basis for $M^{10,1}$ in such a way that the initial rotation $R \in \mathrm{SO}_{9}$ belongs to a given maximal torus of $\mathrm{SO}_{9}$. The canonical embedding $\mathrm{SO}_{8} \subset \mathrm{SO}_{9}$ is such that the maximal tori agree. Therefore we can take $R \in \mathrm{SO}_{8}$ without loss of generality. Moreover we can always choose our basis so that $\pi=e_{0} \wedge e_{1} \wedge e_{3} \wedge \cdots \wedge e_{9}$, and such that $R$, which is parametrised by four angles, is given by the block-diagonal matrix

$$
R(\theta)=\left(\begin{array}{llll}
R_{12}\left(\theta_{1}\right) & & & \\
& R_{34}\left(\theta_{2}\right) & & \\
& & R_{56}\left(\theta_{3}\right) & \\
& & & R_{78}\left(\theta_{4}\right)
\end{array}\right) \in \mathrm{SO}_{8},
$$

\footnotetext{
${ }^{1}$ Strictly speaking it need not be a pure boost: we simply mean that it is not a rotation.
} 
each $R_{j k}(\vartheta)$ being the rotation by an angle $\vartheta$ in the 2-plane spanned by $e_{j}$ and $e_{k}$. The angles $\left(\theta_{i}\right)$ are of course only defined up to Weyl transformations.

Having rotated, we now make a Lorentz transformation normal to the $\mathbb{E}^{8}$ on which this $\mathrm{SO}_{8}$ acts. In other words, we have in effect broken the Lorentz group $\mathrm{SO}_{10,1}^{0}$ down to $\mathrm{SO}_{8} \times \mathrm{SO}_{2,1}^{0}$, where $\mathrm{SO}_{2,1}^{0}$ acts on the three-dimensional space spanned by $e_{0}, e_{9}, e_{\mathrm{t}}$. Because $e_{9}$ is tangent to the brane, boosts along $e_{9}$ do not alter the configuration, so that we can restrict ourselves to those Lorentz transformations in $\mathrm{SO}_{2,1}^{0}$ which do not act trivially on the $e_{\natural}$ direction. The most general such element of $\mathrm{SO}_{2,1}^{0}$ is parametrised by a vector $v=\alpha e_{0}+\beta e_{9}, \alpha, \beta \in \mathbb{R}$,

$$
S(v)=\exp \left(\alpha \Sigma_{0 \natural}+\beta \Sigma_{9 \natural}\right),
$$

where $\Sigma_{\mu \nu}$ are the generators of $\mathfrak{s o}_{2,1}$.

We can distinguish three different types of transformations $S(v)$ depending on whether $v$ is time-like, space-like or null. If $v$ is space-like we are basically doing a rotation in the plane $v \wedge e_{\natural}$. Since $v$ belongs to both $\pi$ and $R \pi$, we can change basis from $\left\{e_{0}, e_{9}, e_{\natural}\right\}$ to $\left\{e_{0}, v /|v|, e_{\natural}\right\}$ and the transformation $L=R(\theta) S(v)$ is simply a rotation in $\mathrm{SO}_{10}$, which was treated in detail in $[9,1]$. If $v$ is time-like, then we can change basis from $\left\{e_{0}, e_{9}, e_{\natural}\right\}$ to $\left\{v /|v|, e_{9}, e_{\natural}\right\}$. $S(v)$ now corresponds to a pure boost in the $e_{\natural}$ direction. We will see below that the transformation $L=R(\theta) S(v)$ will only preserve some supersymmetry if the boost parameter is zero, so that $S(v)=\mathbb{1}$. Therefore this case once again falls into the analysis in [9, 1]. Finally, we consider the case of $v$ null. In this case, $S(v)$ leaves $v$ invariant, and hence corresponds to a null rotation (see, e.g., [10]). As will see, this case will give rise to new supersymmetric configurations.

To summarise, we can always choose basis such that the plane $\pi=$ $e_{0} \wedge e_{1} \wedge e_{3} \wedge \cdots \wedge e_{9}$ and such that the Lorentz transformation $L$ takes the form

$$
L=\left(\begin{array}{l|l}
R & \\
\hline & S
\end{array}\right) \in \mathrm{SO}_{8} \times \mathrm{SO}_{2,1}^{0} \subset \mathrm{SO}_{10,1}^{0},
$$

where $R$ is in the maximal torus defined in (3) and where $S$ is either a rotation in the $e_{9} \wedge e_{\natural}$ plane, a boost in the $e_{\natural}$ direction, or a null rotation. We now proceed to analyse the supersymmetry of a configuration of two branes with tangent planes $\pi$ and $L \pi$, with $\pi$ and $L$ given above.

3.2. Conditions for supersymmetry. We are interested in solving equation (2) simultaneously for the tangent planes $\pi$ and $L \pi$. Let $\widehat{L}$ denote any one of the two possible lifts to $\operatorname{Spin}_{10,1}$ of the Lorentz transformation $L \in \mathrm{SO}_{10,1}^{0}$. Then equation (2) for $L \pi$ can be written 
as follows:

$$
\widehat{L} \cdot \pi \cdot \widehat{L}^{-1} \cdot \varepsilon=\varepsilon .
$$

Up to a sign, $\widehat{L}$ is given by

$$
\widehat{L}=\exp \left(\frac{1}{2} \theta_{1} \Gamma_{12}+\frac{1}{2} \theta_{2} \Gamma_{34}+\frac{1}{2} \theta_{3} \Gamma_{56}+\frac{1}{2} \theta_{4} \Gamma_{78}+\frac{1}{2} \alpha \Gamma_{0 \natural}+\frac{1}{2} \beta \Gamma_{9 \natural}\right),
$$

from which it follows that since $\pi=e_{0} \wedge e_{1} \wedge e_{3} \wedge \cdots \wedge e_{9}$,

$$
\pi \cdot \widehat{L}^{-1}=\widehat{L} \cdot \pi .
$$

Plugging this into (5), and using (2), we find that (5) follows from (2) and

$$
\widehat{L}^{2} \cdot \varepsilon=\varepsilon,
$$

with the same equation resulting for the other possible lift $-\widehat{L}$.

In order to analyse this equation, it is convenient to break up $\widehat{L}$ as in (4), $\widehat{L}=\widehat{R} \widehat{S}$. Then equation (7) becomes

$$
\begin{aligned}
\varepsilon & =\widehat{R}^{2} \cdot \widehat{S}^{2} \cdot \varepsilon \\
& =\exp \left(\theta_{1} \Gamma_{12}+\theta_{2} \Gamma_{34}+\theta_{3} \Gamma_{56}+\theta_{4} \Gamma_{78}\right) \cdot \exp \left(\alpha \Gamma_{0 \natural}+\beta \Gamma_{9 \natural}\right) \cdot \varepsilon .
\end{aligned}
$$

The first exponential expands to

$$
\widehat{R}^{2}=\left(\cos \theta_{1}+\sin \theta_{1} \Gamma_{12}\right) \cdots\left(\cos \theta_{4}+\sin \theta_{4} \Gamma_{78}\right),
$$

which is an element in the maximal torus of $\operatorname{Spin}_{8}$. For the second exponential we have to distinguish three cases:

1. $\alpha^{2}<\beta^{2}$. In this case, let $\gamma=+\sqrt{\beta^{2}-\alpha^{2}}$. The exponential $\overline{\text { becomes }}$

$$
\widehat{S}^{2}=\cos \gamma+\sin \gamma\left(\frac{\alpha}{\gamma} \Gamma_{0 \natural}+\frac{\beta}{\gamma} \Gamma_{9 \natural}\right) .
$$

Notice that in this case, the matrix in parenthesis obeys

$$
\left(\frac{\alpha}{\gamma} \Gamma_{0 \natural}+\frac{\beta}{\gamma} \Gamma_{9 \natural}\right)^{2}=-\mathbb{1},
$$

whence it is a complex structure just like $\Gamma_{12}, \Gamma_{34}, \Gamma_{56}$ and $\Gamma_{78}$ with which it commutes. This means that $\widehat{L}^{2}=\widehat{R}^{2} \widehat{S}^{2}$ belongs to the maximal torus of $\operatorname{Spin}_{10}$. This case was studied in [9] and [1] and we will have nothing more to add here.

2. $\alpha^{2}>\beta^{2}$. In this case, let $\gamma=+\sqrt{\alpha^{2}-\beta^{2}}$. The exponential becomes

$$
\widehat{S}^{2}=\cosh \gamma+\sinh \gamma\left(\frac{\alpha}{\gamma} \Gamma_{0 \natural}+\frac{\beta}{\gamma} \Gamma_{9 \natural}\right) .
$$

Defining $\delta=-\sqrt{-1} \gamma$ and noticing that $\sinh \gamma=\sqrt{-1} \sin \delta$, we can rewrite $\widehat{S}^{2}$ as an imaginary rotation

$$
\widehat{S}^{2}=\cos \delta+\sin \delta\left(\frac{\alpha}{\delta} \Gamma_{0 \natural}+\frac{\beta}{\delta} \Gamma_{9 \natural}\right),
$$


where the matrix in parenthesis is also a complex structure

$$
\left(\frac{\alpha}{\delta} \Gamma_{0 \natural}+\frac{\beta}{\delta} \Gamma_{9 \natural}\right)^{2}=-\mathbb{1} .
$$

This means that formally $\widehat{L}^{2}=\widehat{R}^{2} \widehat{S}^{2}$ belongs to the maximal torus of $\operatorname{Spin}_{10}$, but where one of the angles is pure imaginary. This will allow us to use the results of [9] to treat this case. It was shown in [9] (see also [2, 1]) that this configuration is supersymmetric if and only if the sum of the angles is zero modulo $2 \pi$. In [9] this follows from an explicit calculation which only uses the fact that the four complex structures in $\widehat{R}^{2}$ and the one in $\widehat{S}^{2}$ all commute. Since the angles can now be complex, their sum vanishes (modulo $2 \pi$ ) provided that both the real and imaginary parts of their sum vanish. Therefore $\delta$, being the only imaginary angle, has to vanish, and $\widehat{L}^{2}=\widehat{R}^{2}$ which is a rotation in the maximal torus of $\operatorname{Spin}_{8}$. This therefore reduces to the cases studied before, and will not studied further in this paper.

3. $\alpha^{2}=\beta^{2}$. In this case, the exponential truncates to a linear term:

$$
\widehat{S}^{2}=\mathbb{1}+\alpha \Gamma_{0 \natural}+\beta \Gamma_{9 \natural} .
$$

This case will yield the constructions of new supersymmetric configurations of intersecting branes in motion, and we now turn to its detailed analysis.

3.3. Supersymmetry and null rotations. Last, but not least, we consider Case 3. Now we have

$$
\widehat{L}^{2}=\widehat{R}^{2} \cdot(\mathbb{1}+\widehat{N}),
$$

where $\widehat{N}$ denotes the nilpotent matrix

$$
\widehat{N} \equiv \alpha \Gamma_{0 \natural}+\beta \Gamma_{9 \natural},
$$

obeying $\widehat{N}^{2}=0$. Equation (7) becomes

$$
\left(\widehat{R}^{2}-\mathbb{1}+\widehat{N}\right) \cdot \varepsilon=0
$$

This equation means that $\varepsilon$ lies in the kernel of a linear transformation with a Jordan-Chevalley decomposition consisting of a nilpotent piece $\widehat{N}$ and a semisimple piece $\left(\widehat{R}^{2}-\mathbb{1}\right)$, which commute with each other. It follows that $\varepsilon$ must be annihilated by both pieces simultaneously. Therefore for two branes related by a rotation $R$ together with a null rotation $(\mathbb{1}+N)$ in a perpendicular plane, the conditions for supersymmetry become the following three equations:

$$
\pi \cdot \varepsilon=\varepsilon, \quad \widehat{R}^{2} \cdot \varepsilon=\varepsilon \quad \text { and } \quad \widehat{N} \cdot \varepsilon=0 .
$$

The first two equations are the conditions for two rotated branes to be supersymmetric. They were originally studied in [9] who classified all 
possible configurations. In their nomenclature they correspond to those rotations with four angles or less. In [1] we showed that rotations $\widehat{R}^{2}$ for which these equations have some solutions must belong to the maximal torus of $\operatorname{Spin}_{7}$ (equivalently the maximal torus of its subgroup $\mathrm{SU}_{4}$ ) in $\operatorname{Spin}_{8}$. Such configurations generically preserve a fraction $\nu=\frac{1}{16}$ of the supersymmetry, but one can preserve a larger fraction by specialising to a descending chain of subgroups. The total fraction $\nu$, once we have imposed the third equation in (8), will be further halved, because only half of those spinors which satisfy the first two equations also satisfy the third. Let us see this in more detail.

To understand the third equation, let us introduce the "contracting homotopy" $K=\alpha \Gamma_{0 \natural}-\beta \Gamma_{9 \natural}$, which satisfies

$$
K \cdot \widehat{N}+\widehat{N} \cdot K=4 \alpha^{2} \mathbb{1} .
$$

Applying both sides of the equation to $\varepsilon$ we see that

$$
\widehat{N} \cdot \varepsilon=0 \Longrightarrow \varepsilon=\widehat{N} \cdot(\widehat{K} \cdot \varepsilon),
$$

where we have introduced $\widehat{K} \equiv \frac{1}{4 \alpha^{2}} K$. A similar result holds for spinors which are annihilated by $\widehat{K}$. As a consequence of equation (9), the spinor representation $\Delta$ has a vector space decomposition:

$$
\Delta=\operatorname{ker} \widehat{N} \oplus \operatorname{ker} \widehat{K} \text {, }
$$

and $\widehat{K}$ provides an isomorphism $\operatorname{ker} \widehat{N} \rightarrow \operatorname{ker} \widehat{K}$. In other words, $\operatorname{dim} \operatorname{ker} \widehat{N}=\operatorname{dim} \operatorname{ker} \widehat{K}=16$. Because $\widehat{R}^{2}$ commutes with $\widehat{N}$ and with $\widehat{K}$, it respects and hence refines the decomposition.

We now describe the action of $\widehat{R}^{2}$ on $\Delta$. Because $\widehat{R}^{2}$ belongs to (the maximal torus of) $\operatorname{Spin}_{8}$, we first realise $\Delta$ as a representation of $\mathrm{Spin}_{8} \subset \mathrm{C} \ell_{8}$. As will be shown later, in terms of representation of $\mathrm{C} \ell_{8}$, $\Delta=\Delta^{\prime} \otimes \mathbb{R}^{2}$, where $\Delta^{\prime}$ is the irreducible real representation of $\mathrm{C} \ell_{8}$ and $\mathbb{R}^{2}$ is two copies of the trivial representation. In terms of $\operatorname{Spin}_{8}, \Delta^{\prime}$ breaks up further as $\Delta_{+}^{\prime} \oplus \Delta_{-}^{\prime}$, which are the representations of definite chirality; although we will not need this further decomposition in the sequel.

Under the action of $\widehat{R}^{2}, \Delta^{\prime}$ breaks up as follows:

$$
\Delta^{\prime}=\bigoplus_{\vartheta} n_{\vartheta} \Delta_{\vartheta}^{\prime}
$$

where $\vartheta$ are angles and the multiplicity $n_{\vartheta}$ is a positive integer. For $\vartheta \neq 0, \pi, \widehat{R}^{2}$ restricts to $\Delta_{\vartheta}^{\prime}$ as a $2 \times 2$ matrix of the form

$$
\left(\begin{array}{rr}
\cos \vartheta & \sin \vartheta \\
-\sin \vartheta & \cos \vartheta
\end{array}\right) \text {. }
$$

For $\vartheta=0, \pi, \Delta_{0}^{\prime}$ and $\Delta_{\pi}^{\prime}$ are one-dimensional with $\widehat{R}^{2}$ restricting to \pm 1 respectively. A closer look at the weights of the half-spin representations of $\operatorname{Spin}_{8}$ shows that if a weight appears, then so does its negative; 
whence $n_{0}$ and $n_{\pi}$ are actually even. Applying this to $\Delta$, we have the following decomposition

$$
\Delta=\bigoplus_{\vartheta} 2 n_{\vartheta} \Delta_{\vartheta}^{\prime}
$$

Let $\Delta_{0}$ denote the $2 n_{0}$-dimensional subspace of $\Delta$ defined by those $\varepsilon \in \Delta$ obeying the second equation in (8). As we have just seen, the dimension of $\Delta_{0}$ is divisible by 4 .

Because $\widehat{N}$ and $\widehat{K}$ commute with $\widehat{R}^{2}$, they preserve $\Delta_{0}$. Let $\widehat{N}_{0}$ and $\widehat{K}_{0}$ denote their restrictions to $\Delta_{0}$. Equation (9) again implies that we can decompose

$$
\Delta_{0}=\operatorname{ker} \widehat{N}_{0} \oplus \operatorname{ker} \widehat{K}_{0}
$$

with $\widehat{K}_{0}$ giving an isomorphism ker $\widehat{N}_{0} \rightarrow$ ker $\widehat{K}_{0}$; whence they have the same dimension: $\frac{1}{2}$ the dimension of $\Delta_{0}$.

Similarly, from (6), $\pi$ preserves $\Delta_{0}$ and also this decomposition. Let $\pi_{0}$ denote the restriction of $\pi$ to $\Delta_{0}$. Since $\pi_{0} \cdot \pi_{0}=\mathbb{1}, \pi_{0}$ decomposes $\Delta_{0}$ as

$$
\Delta_{0}=\Delta_{0}^{+} \oplus \Delta_{0}^{-}
$$

and also the subspace $\operatorname{ker} \widehat{N}_{0}$ as

$$
\operatorname{ker} \widehat{N}_{0}=\left(\operatorname{ker} \widehat{N}_{0}\right)^{+} \oplus\left(\operatorname{ker} \widehat{N}_{0}\right)^{-},
$$

in the obvious way. Equation (8) says that $\varepsilon$ belongs to $\left(\operatorname{ker} \widehat{N}_{0}\right)^{+}$, and we would like to compute its dimension. We will show that it is $\frac{1}{4}$ the dimension of $\Delta_{0}$.

To see this consider $\Gamma_{12}$. It commutes with $\widehat{R}^{2}, \widehat{N}$ and $\widehat{K}$ and anticommutes with $\pi$. Therefore it maps $\Delta_{0}^{+}$isomorphically to $\Delta_{0}^{-}$and in particular $\left(\operatorname{ker} \widehat{N}_{0}\right)^{+}$isomorphically to $\left(\operatorname{ker} \widehat{N}_{0}\right)^{-}$. It follows that these latter subspaces have the same dimension: $\frac{1}{2}$ the dimension of ker $\widehat{N}_{0}$. In summary,

$$
\operatorname{dim}\left(\operatorname{ker} \widehat{N}_{0}\right)^{+}=\frac{1}{2} \operatorname{dim} \operatorname{ker} \widehat{N}_{0}=\frac{1}{4} \operatorname{dim} \Delta_{0} .
$$

The second equation in (8) says that $\varepsilon$ belongs to $\Delta_{0}$, whereas the third equation forces it to belong to ker $\widehat{N}_{0}$. The first equation in (8) further constraints $\varepsilon$ to live in $\left(\operatorname{ker} \widehat{N}_{0}\right)^{+}$. In other words, the number of spinors satisfying all three equations in (8) is one half the number satisfying the first two. From the known results [9] about the solutions to the first two equations, we can immediately write down the possible fractions in terms of the codimension of the spatial intersection of the two branes (see [1]): 


\begin{tabular}{|c|l|}
\hline Codimension $d$ & \multicolumn{1}{|c|}{ Fractions $\nu$} \\
\hline \hline 4 & $\frac{1}{32} \rightarrow \frac{1}{16} \rightarrow \frac{3}{32} \rightarrow \frac{1}{8}$ \\
3 & $\frac{1}{16}$ \\
2 & $\frac{1}{8}$ \\
0 & $\frac{1}{4}$ \\
\hline
\end{tabular}

TABLE 1. Fractions of supersymmetry appearing in configurations of two null-rotated M5-branes, in terms of the codimension of the intersection. Arrows indicate progressive specialisation.

3.4. Group-theoretical analysis. We now interpret the above results in terms of group theory, as was done in Part I for the solutions found in [9]. We will be brief because, as we have just seen, the new solutions are obtained by null-rotating some of the supersymmetric configurations consisting of two intersecting M5-branes at angles, and the group theory for those configurations has been discussed in detail in Part I.

Not every intersecting brane configuration can be null rotated to obtain a different supersymmetric configuration. In the notation of [9] we need to restrict ourselves to configurations with at most four angles, whereas in the notation of Part I, the codimension must be at most four.

These configurations were shown in Part I to correspond to subgroups $G \subset \operatorname{Spin}_{8}$ which preserve some spinor. More precisely, $\widehat{R}^{2} \in$ $\mathbb{T}(G)$, the maximal torus of $G$. A list of possible subgroups is displayed in Figure 1. The first row consists of subgroups of $\operatorname{Spin}_{8}$, the second of subgroups of $\operatorname{Spin}_{7}$, the third of $\operatorname{Spin}_{6}$ and the fourth of $\operatorname{Spin}_{4}$. The first row is nothing but the spin series: $\operatorname{Spin}_{7} \supset \operatorname{Spin}_{6} \cong \mathrm{SU}_{4} \supset \operatorname{Spin}_{5} \cong$ $\mathrm{Sp}_{2} \supset \mathrm{Spin}_{4} \cong \mathrm{Sp}_{1} \times \mathrm{Sp}_{1} \supset \operatorname{Spin}_{3} \cong \mathrm{Sp}_{1} \supset \operatorname{Spin}_{2} \cong \mathrm{U}_{1}$.

The two M5-branes are related by $\mathbb{T}(G)$ in the above figure as well as by a null rotation, which forms a subgroup $\mathbb{R} \subset \operatorname{Spin}_{10,1}$. Therefore the branes are $(\mathbb{T}(G) \times \mathbb{R})$-related. As discussed for example in Part I, the groups $G$ relating the branes are subgroups of $\mathrm{SU}_{5}$ or $\mathrm{Spin}_{7}$, which, together with their intersection $\mathrm{SU}_{4}$, are the subgroups of $\mathrm{Spin}_{10}$ which leave a spinor invariant. In the more general setup of this paper, we expect the groups $\mathbb{T}(G) \times \mathbb{R}$ to be subgroups of the isotropy groups of spinors in $\operatorname{Spin}_{10,1}$. In fact, as we shall see in the next section this is indeed the case. The group in question is a non-reductive 30-dimensional Lie subgroup of $\operatorname{Spin}_{10,1}$ isomorphic to $\operatorname{Spin}_{7} \ltimes \mathbb{R}^{9}$, where $\operatorname{Spin}_{7} \subset \operatorname{Spin}_{8}$ leaves the vector representation irreducible, and where $\mathbb{R}^{9}$ acts by null rotations. 


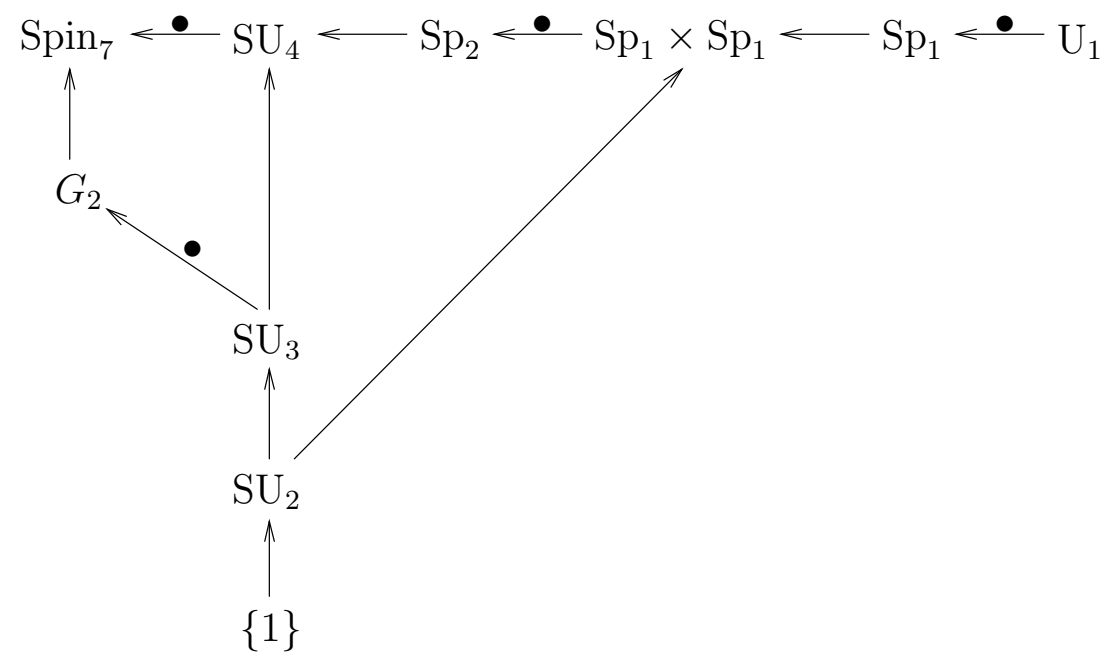

FIGURE 1. Subgroups of $\operatorname{Spin}_{8}$ associated with intersecting brane configurations which can be null-rotated while preserving some supersymmetry. Arrows represent embeddings, and those adorned with a $\bullet$ are such that the maximal tori agree.

\section{Null ROtATIONS AND AN EXOTIC SPINOR ISOTROPY GROUP}

As preparation for our discussion of the multiple intersection problem in the next section, we collect some basic facts about elevendimensional spinors and in particular their 'exotic' isotropy. It will be convenient for some of the calculations to work in a specific realisation for the Clifford algebra $\mathrm{C} \ell_{1,10}$, so we start by discussing this. We then turn our attention to the isotropy subgroup $\left(\operatorname{Spin}_{7} \ltimes \mathbb{R}^{9}\right) \subset \operatorname{Spin}_{10,1}$. The $\mathbb{R}^{9}$ subgroup acts as null rotations and we collect some results about them.

4.1. An explicit real realisation of $\mathrm{C} \ell_{1,10}$. Because as a real associative algebra, $\mathrm{C}_{1,10} \cong \operatorname{Mat}_{32}(\mathbb{R}) \oplus \operatorname{Mat}_{32}(\mathbb{R})$, there are two inequivalent irreducible representations: both real and of dimension 32. Choosing a set of generators $\Gamma_{0}, \Gamma_{1}, \ldots, \Gamma_{9}, \Gamma_{\natural}$ for $\mathrm{C} \ell_{1,10}$, their product $\Gamma_{012 \cdots 9}$ commutes with all $\Gamma_{M}$ and squares to one. Hence by Schur's lemma it is $\pm \mathbb{1}$ on an irreducible representation. We choose $\Delta$ to be the one on which $\Gamma_{012 \ldots 9 \natural}$ takes the value $-\mathbb{1}$. This means that $\Gamma_{0}=\Gamma_{1 \ldots 9 \natural}$, where $\Gamma_{1}, \ldots, \Gamma_{9}, \Gamma_{\natural}$ generate $\mathrm{C} \ell_{0,10}$. The Clifford algebra $\mathrm{C} \ell_{0,10}$ is isomorphic to $\mathrm{C} \ell_{8} \otimes \mathrm{C} \ell_{0,2}$, where the isomorphism is given explicitly as follows in terms of generators. Let $\Gamma_{i}^{\prime}$ for $i=1,2, \ldots, 8$ denote the generators for $\mathrm{C} \ell_{8}$ and let $\Gamma_{1}^{\prime \prime}$ and $\Gamma_{2}^{\prime \prime}$ denote the generators for $\mathrm{C} \ell_{0,2}$.

The $\Gamma_{i}^{\prime}$ can be constructed explicitly in terms of octonions. The construction of the two irreducible representations of $\mathrm{C}_{7}$ in terms of octonions is well known: see, for example, [8]. Let $\left\{o_{i}\right\}, i=1, \ldots, 7$, be 
a set of imaginary octonion units. Then left $L_{i}$ and right multiplication $R_{i}$ by $o_{i}$ on $\mathbb{O}$ define the two inequivalent irreducible representations of the Clifford algebra $\mathrm{C}_{7}$. Either representation can be used in order to build the unique irreducible representation of $\mathrm{C} \ell_{8}$, we choose $L_{i}$. We define

$$
\Gamma_{i}^{\prime}=\left(\begin{array}{cc}
0 & L_{i} \\
L_{i} & 0
\end{array}\right) \quad \text { for } i=1, \ldots, 7 ; \text { and } \quad \Gamma_{8}^{\prime}=\left(\begin{array}{cc}
0 & -\mathbb{1} \\
\mathbb{1} & 0
\end{array}\right) .
$$

This gives rise to a manifestly real 16-dimensional representation.

As associative algebras, $\mathrm{C} \ell_{0,2} \cong \operatorname{Mat}_{2}(\mathbb{R})$, so we can choose a basis

$$
\begin{aligned}
\Gamma_{1}^{\prime \prime} & =\sigma_{1}=\left(\begin{array}{ll}
0 & 1 \\
1 & 0
\end{array}\right) \quad \Gamma_{2}^{\prime \prime}=\sigma_{3}=\left(\begin{array}{cc}
1 & 0 \\
0 & -1
\end{array}\right) \\
\therefore \quad \Gamma_{3}^{\prime \prime} & =\Gamma_{1}^{\prime \prime} \Gamma_{2}^{\prime \prime}=-i \sigma_{2}=\left(\begin{array}{cc}
0 & -1 \\
1 & 0
\end{array}\right) .
\end{aligned}
$$

Then the generators of $\mathrm{C} \ell_{0,10}$ are given by

$$
\begin{aligned}
\Gamma_{i} & =\Gamma_{i}^{\prime} \otimes \Gamma_{3}^{\prime \prime} \quad \text { for } i=1,2, \ldots, 8 \\
\Gamma_{9} & =\mathbb{1} \otimes \Gamma_{1}^{\prime \prime} \\
\Gamma_{\natural} & =\mathbb{1} \otimes \Gamma_{2}^{\prime \prime} \\
\therefore \quad \Gamma_{0} & =\Gamma_{9}^{\prime} \otimes \Gamma_{3}^{\prime \prime},
\end{aligned}
$$

where $\Gamma_{9}^{\prime} \equiv \Gamma_{1}^{\prime} \Gamma_{2}^{\prime} \cdots \Gamma_{8}^{\prime}$. This decomposition induces an isomorphism $\Delta \cong \mathbb{R}^{16} \otimes \mathbb{R}^{2}$, so that we can write our spinors as two-component objects, each component being a sixteen-dimensional real spinor of $\mathrm{C} \ell_{8}$. Therefore in terms of $\mathrm{C} \ell_{8}$ generators we have

$$
\begin{aligned}
\Gamma_{0}=\left(\begin{array}{cc}
0 & -\Gamma_{9}^{\prime} \\
\Gamma_{9}^{\prime} & 0
\end{array}\right) & \Gamma_{i}=\left(\begin{array}{cc}
0 & -\Gamma_{i}^{\prime} \\
\Gamma_{i}^{\prime} & 0
\end{array}\right) \text { for } i=1,2, \ldots, 8 \\
\Gamma_{9}=\left(\begin{array}{ll}
0 & \mathbb{1} \\
\mathbb{1} & 0
\end{array}\right) & \Gamma_{\natural}=\left(\begin{array}{cc}
\mathbb{1} & 0 \\
0 & -\mathbb{1}
\end{array}\right) .
\end{aligned}
$$

The standard basis for the Lie algebra $\mathfrak{s o}_{10,1} \subset \mathrm{C} \ell_{1,10}$ is given by $\Sigma_{M N}=-\frac{1}{2} \Gamma_{M N}$, which in the chosen realisation becomes

$$
\begin{array}{rlrl}
\Sigma_{i j} & =\left(\begin{array}{cc}
\Sigma_{i j}^{\prime} & 0 \\
0 & \Sigma_{i j}^{\prime}
\end{array}\right) & \Sigma_{i 9}=\left(\begin{array}{cc}
\frac{1}{2} \Gamma_{i}^{\prime} & 0 \\
0 & -\frac{1}{2} \Gamma_{i}^{\prime}
\end{array}\right) \\
\Sigma_{i \natural}=\left(\begin{array}{cc}
0 & -\frac{1}{2} \Gamma_{i}^{\prime} \\
-\frac{1}{2} \Gamma_{i}^{\prime} & 0
\end{array}\right) & \Sigma_{0 i}=\left(\begin{array}{cc}
\frac{1}{2} \Gamma_{9}^{\prime} \Gamma_{i}^{\prime} & 0 \\
0 & \frac{1}{2} \Gamma_{9}^{\prime} \Gamma_{i}^{\prime}
\end{array}\right) \\
\Sigma_{09}=\left(\begin{array}{cc}
\frac{1}{2} \Gamma_{9}^{\prime} & 0 \\
0 & -\frac{1}{2} \Gamma_{9}^{\prime}
\end{array}\right) & \Sigma_{0 \natural}=\left(\begin{array}{cc}
0 & -\frac{1}{2} \Gamma_{9}^{\prime} \\
-\frac{1}{2} \Gamma_{9}^{\prime} & 0
\end{array}\right) \\
\Sigma_{9 \natural}=\left(\begin{array}{cc}
0 & \frac{1}{2} \mathbb{1} \\
-\frac{1}{2} \mathbb{1} & 0
\end{array}\right), &
\end{array}
$$

where $\Sigma_{i j}^{\prime}$ are the generators of $\mathfrak{s o}_{8} \subset \mathrm{C} \ell_{8}$. In particular, notice that as mentioned above, the representation $\Delta$ breaks up under $\operatorname{Spin}_{8}$ as 
two copies each of the half-spin representations. In a more traditional language, under the embedding $\operatorname{Spin}_{10,1} \supset \operatorname{Spin}_{8}$,

$$
\underline{\mathbf{3 2}}=2 \underline{\mathbf{8}}_{\mathrm{s}} \oplus 2 \underline{\mathbf{8}}_{\mathrm{c}} \text {. }
$$

4.2. Spinor isotropies. As was done in Part I, in order to construct supersymmetric configurations involving more than two branes-indeed an arbitrary number - we can consider branes which are related by subgroups $G$ of the isotropy group of a spinor. This prompts the following question:

Question 4. Which are the possible subgroups of $\operatorname{Spin}_{10,1}$ which leave invariant a spinor?

This question is intimately linked to the orbit decomposition under $\operatorname{Spin}_{10,1}$ of its spinor representation $\Delta$. One way to study this problem is to define a $\operatorname{Spin}_{10,1}$ invariant function on $\Delta$ which distinguishes the orbits. Bryant [3] defines a quartic polynomial invariant on $\Delta$. This quartic polynomial is nothing but the norm of the vector associated to the spinor. In other words, if $\varepsilon \in \Delta$ we let $v$ denote the vector whose components in the chosen basis are $v_{M}=\bar{\varepsilon} \Gamma_{M} \varepsilon$. Its Minkowskian norm $\eta^{M N} v_{M} v_{N}$ is a quartic polynomial on spinors which is manifestly $\operatorname{Spin}_{10,1}$-invariant. It is possible to show that this norm is negative semi-definite, whence $v$ is either time-like or null. The orbit of a spinor in $\Delta$ for which $v$ is time-like is 31-dimensional and has isotropy $\mathrm{SU}_{5}$. On the other hand, if $v$ is null, the isotropy subgroup is a 30-dimensional nonreductive Lie group which does not act trivially on the time direction. It is a subgroup of the isotropy subgroup of $v$, which for a null vector is isomorphic to $\operatorname{Spin}_{9} \ltimes \mathbb{R}^{9}$. Indeed, as we will see in the next section, the isotropy subgroup of such a spinor is isomorphic to $\left(\operatorname{Spin}_{7} \ltimes \mathbb{R}^{8}\right) \times \mathbb{R}$, where $\operatorname{Spin}_{7}$ acts on $\mathbb{R}^{8}$ according to the half-spin representation. In terms of its action on $\mathbb{M}^{10,1}, \mathrm{Spin}_{7} \subset \mathrm{SO}_{8}$ acts as rotations in some $\mathbb{E}^{8} \subset M^{10,1}$, and $\mathbb{R}^{9}$ acts via null rotations.

4.3. An exotic spinor isotropy group. In this section we describe a certain 30-dimensional non-reductive Lie subgroup $\mathcal{G} \subset \operatorname{Spin}_{10,1}$ which leaves a spinor invariant. We will exhibit its Lie algebra (and hence the Lie group itself) inside the Clifford algebra $\mathrm{C} \ell_{1,10}$ constructed above.

Consider a spinor $\varepsilon$ of the form

$$
\varepsilon=\left(\begin{array}{c}
\psi_{-} \\
0
\end{array}\right),
$$

where $\psi_{-}$is a negative chirality spinor of $\mathrm{C}_{8}$; that is, $\Gamma_{9}^{\prime} \psi_{-}=-\psi_{-}$. It is easy to compute the isotropy subalgebra $\mathfrak{g} \subset \mathfrak{s o}_{10,1}$ of $\varepsilon$ from the explicit form of the generators of $\mathfrak{s o}_{10,1}$. After a little bit of algebra we obtain that the most general element of $\mathfrak{g}$ is given by

$$
\frac{1}{2} a^{i j} \Sigma_{i j}+b^{i} \Sigma_{+i}+c \Sigma_{+\natural}
$$


where $\Gamma_{+}=\Gamma_{0}+\Gamma_{9}, b^{i}$ and $c$ are arbitrary and $a^{i j}$ are such that $\frac{1}{2} a^{i j} \sum_{i j}^{\prime} \in \mathfrak{s o}_{8}$ is in the isotropy subalgebra of $\psi_{-}$. Because $\operatorname{Spin}_{8}$ acts transitively on the unit sphere in both its spinor (as well as the vector) representations, the isotropy subalgebras of every spinor are conjugate, hence isomorphic. This implies that the isotropy subgroup of $\psi_{-}$is a $\operatorname{Spin}_{7}$ subgroup: one which decomposes the negative spinor representation of $\operatorname{Spin}_{8}$ but keeps the vector representation irreducible. This means that $\frac{1}{2} a^{i j} \sum_{i j}$ belong to an $\mathfrak{s o}_{7}$ subalgebra of $\mathfrak{s o}_{10,1}$. Computing the Lie bracket of elements of the form (14), and using that $\Gamma_{+}$squares to zero, we obtain

$$
\mathfrak{g} \cong\left(\mathfrak{s o}_{7} \ltimes \mathbb{R}^{8}\right) \times \mathbb{R},
$$

where $\mathbb{R}^{8}$ is abelian and $\mathfrak{s o}_{7}$ acts on it as a spinor. Notice that $\mathbb{R}$ is in the centre, and that $\mathbb{R}^{8}$ is an abelian ideal, whence this Lie algebra is not reductive. Exponentiating inside $\mathrm{C} \ell_{1,10}$ we obtain a simply-connected 30-dimensional non-reductive Lie subgroup $\mathcal{G} \subset \operatorname{Spin}_{10,1}$ with the following structure

$$
\mathcal{G} \cong \mathbb{R} \times\left(\mathbb{R}^{8} \rtimes \operatorname{Spin}_{7}\right)
$$

4.4. Invariants. We now investigate the action of $\mathcal{G}$ on $\mathbb{M}^{10,1}$. We will see that it acts reducibly yet indecomposably. It is convenient to parametrise $\mathcal{G}$, which topologically is homeomorphic to $\mathbb{R}^{9} \times \operatorname{Spin}_{7}$, as follows:

$$
\mathrm{C} \ell_{1,10} \supset \mathcal{G} \ni g=\exp \left(c_{\mu} \Sigma_{+\mu}\right) \sigma \text {, }
$$

where $\sigma \in \operatorname{Spin}_{7}$ and $\mu=(i, \downarrow)$, where $i$ runs from 1 to 8 . Notice that the exponential only consists of two terms because $\Gamma_{+}^{2}=0$ :

$$
\exp \left(c_{\mu} \Sigma_{+\mu}\right)=1+c_{\mu} \Sigma_{+\mu}=1+\frac{1}{2} c_{\mu} \Gamma_{\mu} \Gamma_{+} .
$$

The composition of group elements follows the standard semidirect product structure:

$$
\exp \left(c_{\mu} \Sigma_{-\mu}\right) \sigma \exp \left(d_{\mu} \Sigma_{-\mu}\right) \tau=\exp \left(\left(c_{\mu}+\sigma \cdot d_{\mu}\right) \Sigma_{-\mu}\right) \sigma \tau,
$$

where $\sigma, \tau \in \operatorname{Spin}_{7}$ and $c_{\mu}, d_{\mu} \in \mathbb{R}^{9}$.

Let $g \in \mathcal{G}$ be as above. Its action on the basis $\left\{e_{M}\right\}$ is given by

$$
\begin{aligned}
g \cdot e_{i} & =\sigma \cdot e_{i}-\sigma^{-1} \cdot c_{i} e_{+} \\
g \cdot e_{9} & =e_{9}+c_{\mu} e_{\mu}-\frac{1}{2}|c|^{2} e_{+} \\
g \cdot e_{\natural} & =e_{\natural}-c_{\natural} e_{+} \\
g \cdot e_{0} & =e_{0}-c_{\mu} e_{\mu}+\frac{1}{2}|c|^{2} e_{+} \\
\therefore \quad g \cdot e_{+} & =e_{+} \\
\therefore \quad g \cdot e_{-} & =e_{-}-2 c_{\mu} e_{\mu}+|c|^{2} e_{+} .
\end{aligned}
$$

Notice that for nonzero $c_{\mu}$ exactly one null direction is left invariant, so that the transformation is a null rotation. From these formulae 


\begin{tabular}{|c|l|}
\hline Degree & \multicolumn{1}{|c|}{ Invariant forms } \\
\hline \hline 0 & 1 \\
1 & $e_{+}^{*}$ \\
2 & $e_{+}^{*} \wedge e_{\natural}^{*}$ \\
5 & $e_{+}^{*} \wedge \Omega$ \\
6 & $e_{+}^{*} \wedge e_{\natural}^{*} \wedge \Omega$ \\
9 & $e_{+}^{*} \wedge \operatorname{vol}_{8}=e_{+}^{*} \wedge e_{1}^{*} \wedge \cdots \wedge e_{8}^{*}$ \\
10 & $e_{+}^{*} \wedge e_{\natural}^{*} \wedge \operatorname{vol}_{8}$ \\
11 & $\operatorname{vol}_{10,1}=e_{0}^{*} \wedge e_{1}^{*} \wedge \cdots \wedge e_{\natural}^{*}$ \\
\hline
\end{tabular}

TABLE 2. $\mathcal{G}$-invariant forms. Here $e_{M}^{*}$ are canonical dual bases to $e_{M}, \Omega$ is the Cayley form, and $\operatorname{vol}_{8}$ and $\operatorname{vol}_{10,1}$ are the eight- and eleven-dimensional volume forms, respectively.

above one can determine the space of $\mathcal{G}$-invariant forms. The results are summarised in Table 2.

Decomposing the spinor representation $\Delta$ under $\operatorname{Spin}_{7}$, there are precisely two linearly independent $\operatorname{Spin}_{7}$-invariant spinors. The null rotations in $\mathbb{R}^{9}$ preserve only one of them. Therefore $\mathcal{G}$ leaves invariant exactly one spinor (up to scale) - the spinor $\varepsilon$ in (13), where $\psi_{-}$is the $\operatorname{Spin}_{7}$-invariant spinor in that representation. This means that any configuration of $m \mathrm{M}$-branes whose tangent planes are $\mathcal{G}$-related will be supersymmetric, provided that $\varepsilon$ belongs to $\Delta(\pi)$ in the first place.

We conclude this section with a useful fact about null rotations.

Lemma 1. If a spinor in $\Delta$ is annihilated by a (nontrivial) null rotation, then it is annihilated by all null rotations.

Proof. Let $\varepsilon=(\psi \chi)^{t}$ be a spinor in $\Delta$, and let $N=c_{\mu} \Sigma_{+\mu}$ be an infinitesimal null rotation. In terms of the above realisation, we have

$$
N=\left(\begin{array}{cc}
-c_{i} \Gamma_{i}^{\prime} \Pi_{+} & c_{\natural} \Pi_{-} \\
-c_{\natural} \Pi_{+} & c_{i} \Gamma_{i}^{\prime} \Pi_{-}
\end{array}\right),
$$

where $\Pi_{ \pm}=\frac{1}{2}\left(\mathbb{1} \pm \Gamma_{9}^{\prime}\right)$ are the chiral projectors for $\mathrm{C} \ell_{8}$. Therefore $N \cdot \varepsilon=0$ if and only if

$$
c_{\natural} \chi_{-}=c_{i} \Gamma_{i}^{\prime} \psi_{+} \quad \text { and } \quad c_{\natural} \psi_{+}=c_{i} \Gamma_{i}^{\prime} \chi_{-} .
$$

Iterating these equations, we see that

$$
|c|^{2} \psi_{+}=0 \quad \text { and } \quad|c|^{2} \chi_{-}=0 .
$$


Therefore either $c_{\mu}=0$ or $\psi_{+}=\chi_{-}=0$, in which case (16) is satisfied for all $c_{\mu}$. In other words, the kernel of any nontrivial infinitesimal null rotation $c_{\mu} \Sigma_{+\mu}$ coincides with the kernel of $\Gamma_{+}$.

4.5. More on vectors and spinors. In this section we collect one final result we shall need in order to treat the multiple intersection problem. We state the result in a little bit more generality than is needed.

Proposition 1. Let $\varepsilon \in \Delta( \pm \pi)$ for a fixed plane $\pi$. Then the vector $v$ associated to $\varepsilon$ lies in $\pi$.

Proof. Let $\pi$ be a nondegenerate plane and $\pi^{\perp}$ its orthocomplement. Any vector $v$ splits uniquely as $v_{\top}+v_{\perp}$, where $v_{\top} \in \pi$ and $v_{\perp} \in \pi^{\perp}$. It is easy to show that $v_{\top} \cdot \pi=-\pi \cdot v_{\top}$ and that $v_{\perp} \cdot \pi=\pi \cdot v_{\perp}$. Let us introduce projectors $\mathbb{P}_{ \pm}=\frac{1}{2}(\mathbb{1} \pm \pi)$. Let $\varepsilon$ belong to $\Delta( \pm \pi)$, and let us compute the components $\bar{\varepsilon} \cdot v \cdot \varepsilon$ of the vector $v$. From the properties of the charge conjugation $C$ in $C \ell_{1,10}$,

$$
v^{t} \cdot C=-C \cdot v \text {, }
$$

we deduce that

$$
\pi \cdot C=-C \cdot \pi \Longrightarrow \mathbb{P}_{ \pm} \cdot C=C \cdot \mathbb{P}_{\mp} .
$$

Therefore, since $\mathbb{P}_{ \pm} \cdot \varepsilon=\varepsilon$,

$$
\begin{aligned}
\bar{\varepsilon} \cdot v \cdot \varepsilon & =\overline{\mathbb{P}_{ \pm} \cdot \varepsilon} \cdot v \cdot \varepsilon \\
& =\bar{\varepsilon} \cdot \mathbb{P}_{\mp} \cdot\left(v_{\top}+v_{\perp}\right) \cdot \varepsilon \\
& =\bar{\varepsilon} \cdot\left(v_{\top} \cdot \mathbb{P}_{ \pm}+v_{\perp} \cdot \mathbb{P}_{\mp}\right) \cdot \varepsilon \\
& =\bar{\varepsilon} \cdot v_{\top} \cdot \varepsilon
\end{aligned}
$$

so that $v=v_{\top}$.

\section{Multiple intersections}

In this section we discuss multiple intersections. The idea of the construction of supersymmetric intersections of branes is very simple. Suppose that two coincident branes have tangent plane $\pi$. This configuration preserves one half of the supersymmetry, corresponding to the asymptotic values $\varepsilon$ which belong to $\Delta(\pi)$-i.e., which satisfy equation (2). Now let $L$ denote any Lorentz transformation in $\mathrm{SO}_{10,1}^{0}$, and let $\widehat{L}$ denote a lift to $\operatorname{Spin}_{10,1}$. If the lift can be chosen to lie in the isotropy subgroup of the spinor, so that

$$
\widehat{L} \cdot \varepsilon=\varepsilon,
$$

then the Lorentz-transformed brane $L \pi$ also satisfies

$$
L \pi \cdot \varepsilon=\varepsilon .
$$

This means that the brane configuration with tangent planes $\pi$ and $L \pi$ is supersymmetric. We must therefore consider subgroups $G$ of $\operatorname{Spin}_{10,1}$ 
which leave invariant a number of spinors in $\Delta(\pi)$. Then these spinors will also belong to $\Delta(g \pi)$ for all $g \in G$. The problem is therefore to classify all such subgroups $G$ and compute the fraction of the supersymmetry which is preserved by a generic configuration consisting of $G$-related branes.

5.1. G-relatedness. To make this precise, let us adapt the definition of $G$-relatedness given in Part I to our more general situation. Let $G\left((5,1), M^{10,1}\right)$ denote the grassmannian of oriented time-oriented $(5,1)$-planes in $M^{10,1}$. It is acted on transitively by $\mathrm{SO}_{10,1}^{0}$ with isotropy $\mathrm{SO}_{5,1}^{0} \times \mathrm{SO}_{5}$. A given subgroup $G \subset \operatorname{Spin}_{10,1}$ acts on $G\left((5,1), M^{10,1}\right)$ by restricting the action of $\mathrm{SO}_{10,1}^{0}$ to the subgroup to which $G$ gets mapped under the canonical covering map $\operatorname{Spin}_{10,1} \rightarrow \mathrm{SO}_{10,1}^{0}$. We can therefore consider the decomposition of the grassmannian into $G$-orbits.

Definition 1. Let $G \subset \operatorname{Spin}_{10,1}$ and let $\left\{\pi_{i}\right\}$ be $m$ oriented timeoriented $(5,1)$-planes in $M^{10,1}$. We say that they are $G$-related, if they all lie in the same $G$-orbit and furthermore $G$ is the smallest such subgroup of $\operatorname{Spin}_{10,1}$.

In Part I we analysed in detail the case $G \subset \operatorname{Spin}_{10}$. In [2] we proved that a configuration of $m$ static intersecting branes is supersymmetric if and only if the tangent planes are $G$-related, where $G \subset \mathrm{SU}_{5}$ or $G \subset \operatorname{Spin}_{7}$ or both, whence $G \subset \mathrm{SU}_{4}$. This $\operatorname{Spin}_{7}$ subgroup is in fact the intersection with $\operatorname{Spin}_{10}$ of the exotic isotropy subgroup $\left(\operatorname{Spin}_{7} \ltimes\right.$ $\left.\mathbb{R}^{9}\right) \subset \operatorname{Spin}_{10,1}$. Indeed, as we will see presently, a configuration of $m$ intersecting branes in motion is supersymmetric if and only if their tangent planes are $G$-related, where $G=K \ltimes N$ and where $K \subset \operatorname{Spin}_{7}$ and $N \subset \mathbb{R}^{9}$. In fact, we will see that every supersymmetric intersection of M5-branes in motion will correspond to a configuration of Cayley planes in an eight-dimensional euclidean subspace of $M^{10,1}$, which have been null-rotated in the remaining three dimensions.

In Part I we also proved a lower bound for the fraction $\nu$ of the supersymmetry preserved by a configuration of $G$-related branes in terms of the action of the group $G \subset \operatorname{Spin}_{10}$ in the spinor representation $\Delta$. We also conjectured that this lower bound was in fact saturated. In the present case, where $G \subset \mathcal{G}$, the situation is more complicated. Clearly the fraction $\nu$ of the supersymmetry which is preserved by a configuration of $G$-related planes will be larger than or equal to the fraction $\nu_{G}$ defined by

$$
32 \nu_{G}=\operatorname{dim}\left(\Delta(\pi) \cap \Delta^{G}\right),
$$

where $\Delta^{G}$ is the space of $G$-invariant spinors. For $G \subset \operatorname{Spin}_{10}$, we proved in Part I that $\operatorname{dim}\left(\Delta(\pi) \cap \Delta^{G}\right)=\frac{1}{2} \operatorname{dim} \Delta^{G}$; but for $G$ containing null rotations, this is not always the case. Let us analyse this now, and in so doing reduce the problem to one concerning 4-planes in eight dimensions. 
5.2. An equivalent eight-dimensional problem. It is not hard to show that the supersymmetric configurations of intersecting branes in motion consist of null rotated Cayley planes. In fact, suppose that we consider $G$-related planes where $G=K \ltimes N \subset \operatorname{Spin}_{7} \ltimes \mathbb{R}^{9}$, where $N$ is not the trivial group. Let $\pi$ be one of the planes, and let $v$ denote an $N$ invariant null vector. By the Proposition, $v$ belongs to $\pi$. Because $\pi$ is nondegenerate, $\pi$ also contains a complementary null vector $v^{\prime}$, whence we can write $\pi=v \wedge v^{\prime} \wedge \zeta$ where $\zeta$ is a 4 -plane in $\operatorname{span}\left\{v, v^{\prime}\right\}^{\perp} \cong \mathbb{E}^{9}$. Now, $\operatorname{Spin}_{7}$ leaves a direction $u$ in this $\mathbb{E}^{9}$ fixed. Therefore $K \subset \operatorname{Spin}_{7}$ moves $\zeta$ only in an eight-dimensional subspace $\operatorname{span}\left\{v, v^{\prime}, u\right\}^{\perp} \cong \mathbb{E}^{8}$.

We can see this a little bit more explicitly. We want to study $\Delta^{G} \cap$ $\Delta(\pi)$ and compute its dimension in terms of $G$, in the case where $G=$ $K \ltimes N \subset \operatorname{Spin}_{7} \ltimes \mathbb{R}^{9}$. We take $\pi=e_{+} \wedge e_{-} \wedge \zeta$, with $\zeta=e_{1} \wedge e_{3} \wedge e_{5} \wedge e_{7}$. By the Proposition, the null vector associated to this null rotation belongs to $\pi$. Using the Lorentz invariance on $\pi$, we can assume that it is $e_{+}$. Since $G$ contains a nontrivial null rotation, we can first consider the subspace $\Delta^{\prime} \subset \Delta$ defined by

$$
\Delta^{\prime}=\left\{\varepsilon \in \Delta \mid \Gamma_{+} \cdot \varepsilon=0\right\} .
$$

By (the proof of) the Lemma, $\Delta^{\prime}=\Delta^{N}$. It is a 16-dimensional real subspace of $\Delta$. $K$ preserves $\Delta^{\prime}$ because it is contained in $\operatorname{Spin}_{8}$, which commutes with $\Gamma_{+}$. Under $\operatorname{Spin}_{8}, \Delta^{\prime}$ breaks up as two irreducible representations: one of each chirality. In fact, from the proof of the Lemma, it follows that in the chosen realisation, a spinor $\varepsilon$ belongs to $\Delta^{\prime}$ if and only if it has the form

$$
\varepsilon=\left(\begin{array}{l}
\psi_{-} \\
\chi_{+}
\end{array}\right)
$$

From the explicit expression for $\pi, \varepsilon \in \Delta(\pi)$ if and only if

$$
\zeta \cdot \psi_{-}=\psi_{-} \quad \text { and } \quad \zeta \cdot \chi_{+}=\chi_{+} .
$$

In other words, if we make a spinor $\varphi=\left(\psi_{-} \chi_{+}\right)^{t}$ of $\mathrm{C} \ell_{8}$, then $\varepsilon \in \Delta(\pi)$ if and only if $\varphi \in \Delta^{\prime}(\zeta)$ in the obvious notation. In other words, the subspaces $\Delta^{G} \cap \Delta(\pi)$ and $\left(\Delta^{\prime}\right)^{K} \cap \Delta^{\prime}(\zeta)$ of $\Delta$ agree.

It is therefore possible to work with $\Delta^{\prime}$ abstractly as the irreducible representation of $\mathrm{C}_{8}$ and to consider 4 -planes $\zeta$ in $\mathbb{E}^{8}$. As shown in [8] (see also $[7,4,2]$ ), a 4 -plane $\zeta$ in $\mathbb{E}^{8}$ which satisfies

$$
\zeta \cdot \varphi=\varphi,
$$

is a Cayley plane; that is, it is calibrated by the Cayley form built out of $\varphi$ by squaring. In other words, the $G$-related planes are $K$-related Cayley planes which are then null-rotated by $N$.

The most generic situation results from $K=\operatorname{Spin}_{7}$ and $N=\mathbb{R}^{9}$. Then we simply have null-rotated Cayley planes. This is a 17-dimensional orbit inside the 30-dimensional grassmannian of $(5,1)$-planes. Such configurations will generically preserve $\frac{1}{32}$ of the supersymmetry, 
because there is at most one spinor in $\Delta^{\prime}$ which is left invariant by

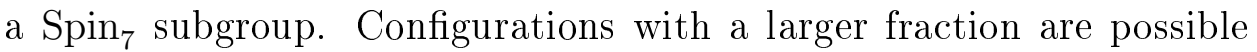
provided that we restrict to planes which live in progressively smaller subspaces of the grassmannian. If $\cup_{i=1}^{m} \zeta_{i}$ are $K$-related, then the resulting brane configuration preserves a fraction $\nu$ of the supersymmetry which obeys $\nu \geq \nu_{K}$, where $\nu_{K}$ is given by

$$
\nu_{K}=\frac{1}{32} \operatorname{dim}\left(\Delta^{\prime}(\zeta) \cap\left(\Delta^{\prime}\right)^{K}\right) .
$$

In Part I we showed that the group-theoretical fraction was directly related to the dimension of group invariant spinors. In the case of nonstatic branes treated here, the dependence of $\nu_{K}$ on $K$ is more subtle, as we now explain.

5.3. The group-theoretical fraction $\nu_{K}$. We would like to compute the dimension of the subspace $\Delta(\pi) \cap \Delta^{G} \subset \Delta$, where $G=K \ltimes N$. In the previous section we saw how this computation can be rephrased in terms of $K$-related Cayley planes in eight dimensions. This was achieved by first taking care of the null rotations and thus reducing the problem to one in lower dimension. Here we will invert the order and first take care of the $K$-invariance. Therefore let $\Delta^{K} \subset \Delta$ denote the space of $K$-invariant spinors in $\Delta . K \subset \operatorname{Spin}_{8}$ and, as we saw in (12), $\Delta$ breaks up under $\operatorname{Spin}_{10,1} \supset \operatorname{Spin}_{8}$ as two copies of each of the half-spin representations. Therefore $\Delta^{K}$ is even-dimensional. As shown in (the proof of) the Lemma, $N$-invariant spinors are those spinors which are annihilated by $\Gamma_{+}$. Because $\Gamma_{+}$is $K$-invariant, $\Gamma_{+}$maps $\Delta^{K}$ to itself. By the same token, so does $\Gamma_{-}$. Because

$$
\Gamma_{+} \Gamma_{-}+\Gamma_{-} \Gamma_{+}=-4 \mathbb{1},
$$

reasons similar to those from which we deduced (10) also yield the following decomposition of $\Delta^{K}$ :

$$
\Delta^{K}=\Delta_{+}^{K} \oplus \Delta_{-}^{K}
$$

where $\Delta_{ \pm}^{K}=\Delta^{K} \cap \operatorname{ker} \Gamma_{ \pm}=\Gamma_{ \pm} \Delta^{K}$. Similarly, it follows that $\Delta_{ \pm}^{K}$ are isomorphic subspaces, the isomorphisms being given by $\Gamma_{ \pm}$. In particular, $\operatorname{dim} \Delta_{ \pm}^{K}=\frac{1}{2} \operatorname{dim} \Delta^{K}$. Because $\Delta^{G}=\Delta_{+}^{K}$, we see that

$$
\operatorname{dim} \Delta^{G}=\frac{1}{2} \operatorname{dim} \Delta^{K}
$$

Now we intersect $\Delta^{G}$ with $\Delta(\pi)$. Let $\left(\Delta^{K}\right)^{ \pm}=\Delta^{K} \cap \Delta( \pm \pi)$. Similarly we can define $\left(\Delta_{ \pm}^{K}\right)^{ \pm}$in the obvious way, where the signs are not correlated. This gives rise to a fourfold decomposition of $\Delta^{K}$ :

$$
\Delta^{K}=\left(\Delta_{+}^{K}\right)^{+} \oplus\left(\Delta_{+}^{K}\right)^{-} \oplus\left(\Delta_{-}^{K}\right)^{+} \oplus\left(\Delta_{-}^{K}\right)^{-} .
$$

By the Proposition, $e_{ \pm}$belong to $\pi$, whence $\Gamma_{ \pm} \cdot \pi=-\pi \cdot \Gamma_{ \pm}$. This means that $\Gamma_{ \pm}$restrict to isomorphisms

$$
\Gamma_{ \pm}:\left(\Delta_{\mp}^{K}\right)^{+} \rightarrow\left(\Delta_{ \pm}^{K}\right)^{-} \quad \text { and } \quad \Gamma_{ \pm}:\left(\Delta_{\mp}^{K}\right)^{-} \rightarrow\left(\Delta_{ \pm}^{K}\right)^{+} .
$$


Letting $d_{ \pm}^{ \pm}=\operatorname{dim}\left(\Delta_{ \pm}^{K}\right)^{ \pm}$, we have that $d_{+}^{+}=d_{-}^{-}$and that $d_{+}^{-}=d_{-}^{+}$, whence

$$
\operatorname{dim} \Delta^{K}=2 d_{+}^{+}+2 d_{+}^{-} .
$$

In other words, since $\left(\Delta(\pi) \cap \Delta^{G}\right)=\left(\Delta_{+}^{K}\right)^{+}$, its dimension is given by

$$
d_{+}^{+}=\frac{1}{2} \operatorname{dim} \Delta^{K}-d_{+}^{-} \leq \frac{1}{2} \operatorname{dim} \Delta^{K} .
$$

It will be convenient in what follows to introduce a rational number $\varrho_{K}$ defined implicitly by

$$
d_{+}^{+}=\varrho_{K} \operatorname{dim} \Delta^{K} .
$$

In contrast with the case of static branes, where $G \subset \operatorname{Spin}_{10}$, we cannot compute $d_{+}^{+}$in a uniform manner, for it seems to depend on other properties of the group besides its invariants on the spinor representation; that is, the ratio $\varrho_{K}$ depends nontrivially on $K$. For example, we saw already that for $m=2$ branes, $d_{+}^{+}=d_{-}^{+}$, whence $\varrho_{K}=\frac{1}{4}$. On the other hand, in some explicit examples we have computed (and which will be discussed briefly below) we also find cases in which $d_{+}^{-}=0$ so that $\varrho_{K}=\frac{1}{2}$, and even some cases for which $\varrho_{K}$ takes less obvious values $\frac{1}{8}, \frac{1}{6}, \frac{1}{5}, \frac{3}{10}, \frac{1}{3}$, and $\frac{3}{8}$. This seems to indicate the need for a case-by-case analysis. We now discuss some explicit examples.

5.4. Some examples and their geometries. In this section we list some examples of $G$-related branes in motion which we have worked out explicitly. These examples are summarised in Table 3. Many of the necessary calculations have been performed infinitesimally (i.e., using their Lie algebras) using Mathematica. ${ }^{2}$

To simplify the table we have used the equivalent eight-dimensional description of the branes in motion as Cayley planes which have been null-rotated. Therefore for branes in motion which are $G$-related, where $G=K \ltimes N$, we have listed the group $K$ together with its codimension, fraction and the ratio $\varrho_{K}$ defined in equation (5.3). We have also listed the isotropy subgroup $H \subset K$ of the reference 4-plane $\zeta$, and the geometry of the resulting grassmannian $K / H$. This is a sub-grassmannian of the Cayley grassmannian, whence it does not take into account the null rotations. The true grassmannian of $G$-related planes is now a homogeneous bundle over $K / H$ with fibre $\mathbb{R}^{5}$, where the $\mathbb{R}^{5}$ factor corresponds to those null rotations defined by vectors in the orthogonal plane $\pi^{\perp}$. Notice that the dependence of $\nu_{K}$ on the subgroup $K$ is subtle, since we find different values of $\varrho_{K}$ for different yet isomorphic subgroups $K$.

Notice also that all the geometries which appear have already been discussed in Part I and hence will not be discussed further here, except for two brief remarks. First, we would like to bring to the attention

\footnotetext{
${ }^{2}$ Details of the calculations can be obtained by email from the authors. They will be made public via our web pages at a later date.
} 


\begin{tabular}{|c|c|c|c|c|c|}
\hline $\begin{array}{c}\text { Codim. } \\
d\end{array}$ & $\begin{array}{c}\text { Group } \\
K\end{array}$ & $\begin{array}{c}\text { Fraction } \\
\nu_{K}\end{array}$ & $\begin{array}{c}\text { Ratio } \\
\varrho_{K}\end{array}$ & $\begin{array}{c}\text { Isotropy } \\
H\end{array}$ & $\begin{array}{c}\text { Geometry } \\
K / H\end{array}$ \\
\hline 4 & $\begin{array}{c}\mathrm{Spin}_{7} \\
\mathrm{SU}_{4} \\
\mathrm{Sp}_{2} \\
\mathrm{Sp}_{1} \times \mathrm{Sp}_{1} \\
\mathrm{SU}_{4} \\
\mathrm{Sp}_{2} \\
\mathrm{Sp}_{1} \times \mathrm{Sp}_{1} \\
\mathrm{Sp}_{1} \\
\mathrm{Sp}_{2} \\
\mathrm{Sp}_{1} \times \mathrm{Sp}_{1} \\
\mathrm{Sp}_{1} \\
\mathrm{U}_{1}\end{array}$ & $\begin{array}{l}\frac{1}{32} \\
\frac{1}{32} \\
\frac{1}{32} \\
\frac{1}{32} \\
\frac{1}{16} \\
\frac{1}{16} \\
\frac{1}{16} \\
\frac{1}{16} \\
\frac{3}{32} \\
\frac{3}{32} \\
\frac{3}{32} \\
\frac{3}{32}\end{array}$ & $\begin{array}{l}\frac{1}{2} \\
\frac{1}{4} \\
\frac{1}{6} \\
\frac{1}{8} \\
\frac{1}{2} \\
\frac{1}{3} \\
\frac{1}{4} \\
\frac{1}{5} \\
\frac{1}{2} \\
\frac{3}{8} \\
\frac{3}{10} \\
\frac{1}{4}\end{array}$ & $\begin{array}{c}\left(\mathrm{SU}_{2}\right)^{3} / \mathbb{Z}_{2} \\
\mathrm{SO}_{4} \\
\mathrm{U}_{2} \\
\mathrm{Sp}_{1} \\
\mathrm{~S}\left(\mathrm{U}_{2} \times \mathrm{U}_{2}\right) \\
\mathrm{U}_{2} \\
\mathrm{U}_{1} \times \mathrm{U}_{1} \\
\mathrm{U}_{1} \\
\mathrm{Sp}_{1} \times \mathrm{Sp}_{1} \\
\mathrm{Sp}_{1} \\
\mathrm{U}_{1} \\
\{1\}\end{array}$ & $\begin{array}{c}\text { Cayley } \\
\text { SLAG }_{4} \\
\mathbb{C L A G}_{2} \\
(3,1) \text { in [5] } \\
\mathbb{C}_{2} \\
\mathbb{C L A G}_{2} \\
\mathbb{C}_{1} \times \mathbb{C}_{1} \\
(3,2) \text { in }[5] \\
\mathrm{H}_{1} \\
(3,1) \text { in }[5] \\
(3,2) \text { in }[5] \\
(3,3) \text { in }[5]\end{array}$ \\
\hline 3 & $\begin{array}{c}G_{2} \\
\mathrm{SU}_{3}\end{array}$ & $\begin{array}{l}\frac{1}{16} \\
\frac{1}{16}\end{array}$ & $\begin{array}{l}\frac{1}{2} \\
\frac{1}{4}\end{array}$ & $\begin{array}{l}\mathrm{SO}_{4} \\
\mathrm{SO}_{3}\end{array}$ & $\begin{array}{l}\text { Associative } \\
\text { SLAG }_{3}\end{array}$ \\
\hline 2 & $\begin{array}{l}\mathrm{SU}_{3} \\
\mathrm{SU}_{2}\end{array}$ & $\begin{array}{l}\frac{1}{8} \\
\frac{1}{8}\end{array}$ & $\begin{array}{l}\frac{1}{2} \\
\frac{1}{4}\end{array}$ & $\begin{array}{c}\mathrm{S}\left(\mathrm{U}_{2} \times \mathrm{U}_{1}\right) \\
\mathrm{SO}_{2}\end{array}$ & $\begin{array}{c}\mathbb{C}_{1} \\
\mathbb{C}_{1} \text { or } \mathrm{SLAG}_{2}\end{array}$ \\
\hline
\end{tabular}

TABLE 3. Some of the geometries associated with supersymmetric configurations of multiply intersecting branes in motion. The table has been compiled in terms of the equivalent eight-dimensional problem discussed in Section 5.2.

of the reader the fact that in this table, and in contrast with the similar table in Part I, isomorphic subgroups $K$ giving rise to isomorphic geometries now yield configurations with different fractions. This does not mean that the dimension of the subspace of $K$-invariant spinors is different, for this only depends on the isomorphism class of $K$. Instead, as mentioned above, it is the intersection of this subspace with $\Delta(\pi)$ which depends subtly on $K$. Finally, let us remark that most of the entries in the table distinguished by having $\varrho_{K} \neq \frac{1}{2}$, correspond to different subgroups than the ones giving rise to the same geometries in the similar table in Part I. 


\section{Summary And Outlook}

In this paper we have obtained new supersymmetric configurations of intersecting branes, consisting of branes which are in relative motion to each other. This work completes the classification of supersymmetric configurations of two intersecting M5-branes, started by Ohta \& Townsend in [9]. As shown in Part I for the static configurations and in the present paper for the rest, each configuration is characterised by (the maximal torus of) a subgroup $G$ of $\operatorname{Spin}_{10,1}$ contained in the isotropy of a spinor. The fraction of the supersymmetry which is preserved is given by $\frac{1}{32}$ times the dimension of the subspace of $\Delta$ consisting of those spinors invariant under $\mathbb{T}(G)$, or when $G=K \ltimes N$, under $\mathbb{T}(K) \times \mathbb{R}$. These results are summarised in Table 4 .

\begin{tabular}{|c|c|c|}
\hline \multirow{2}{*}{$\begin{array}{c}\text { Fraction } \\
\nu\end{array}$} & \multicolumn{2}{|c|}{ Rotation subgroups for } \\
\hline & static branes & branes in motion \\
\hline$\frac{1}{32}$ & $\mathrm{SU}_{5}$ & $\operatorname{Spin}_{7} \stackrel{\circ}{=} \mathrm{SU}_{4}$ \\
\hline$\frac{1}{16}$ & $\mathrm{SU}_{2} \times \mathrm{SU}_{3} \quad \mathrm{Spin}_{7} \stackrel{\circ}{=} \mathrm{SU}_{4}$ & $\mathrm{Sp}_{2} \stackrel{\circ}{=}\left(\mathrm{Sp}_{1}\right)^{2} \quad G_{2} \stackrel{\circ}{=} \mathrm{SU}_{3}$ \\
\hline$\frac{3}{32}$ & $U_{1} \times \mathrm{SU}_{2}$ & $\mathrm{Sp}_{1} \stackrel{\circ}{=} U_{1}$ \\
\hline$\frac{1}{8}$ & 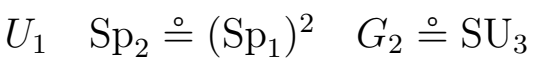 & $\mathbb{Z}_{2} \quad \mathrm{SU}_{2}$ \\
\hline$\frac{5}{32}$ & $\mathbb{Z}_{6}$ & \\
\hline$\frac{3}{16}$ & $\mathrm{Sp}_{1} \stackrel{\circ}{=} \mathrm{U}_{1}$ & \\
\hline$\frac{1}{4}$ & $\mathbb{Z}_{2} \quad \mathrm{SU}_{2}$ & $\{1\}$ \\
\hline$\frac{1}{2}$ & $\{1\}$ & \\
\hline
\end{tabular}

TABLE 4. All possible supersymmetric configurations of two M5-branes, and the corresponding subgroups of $\operatorname{Spin}_{10,1}$. In the case of branes in motion, only the rotation subgroup has been indicated - the null rotations remaining implicit. The relation $\stackrel{\circ}{=}$ denotes subgroups having the same maximal torus. Some finite groups discussed briefly in Part I have been included for completeness.

One can construct each of these configurations starting with two coincident fivebranes and rotate by an element of the maximal torus of a subgroup $G$ of $\operatorname{Spin}_{10}$. If $G$ is actually contained in $\operatorname{Spin}_{8}$ then one can also perform a null-rotation in the $(2+1)$-dimensional subspace left invariant by $\operatorname{Spin}_{8}$. This gives rise to configurations in which the branes are moving relative to each other.

The classification of multiple intersecting brane configurations is more complicated and a complete solution is still lacking. In $[2,1]$ 
as well as in the present paper, we have shown that a configuration preserves some supersymmetry provided that the branes are $G$-related where $G$ is contained in the isotropy of a spinor which obeys equation (2) relative to one of the branes. The possible groups $G$ fall into two classes, depending on whether $G$ is contained or not in $\operatorname{Spin}_{10}$. If $G \subset \operatorname{Spin}_{10}$ then $G$-related branes are static relative to each other since they all share the same time-like direction. If $G$ is not contained in $\operatorname{Spin}_{10}$ we have shown that $G$ contains null rotations, so that $G=K \ltimes N$, where $K \subset \operatorname{Spin}_{8}$ and $N$ contains null rotations. Configurations of $G$-related fivebranes can be understood as $K$-related euclidean fourbranes in eight dimensions which have then been null rotated. These configurations share the same null direction. In Part I we restricted ourselves (following [9]) to static brane configurations and derived a (conjecturally exact) lower bound $\nu_{G}$ for the fraction $\nu$ of the supersymmetry preserved by a configurationof $G$-related branes with $G \subset \operatorname{Spin}_{10}$, in terms of the dimension of the space of $G$-invariant spinors. For $G=K \ltimes N$ containing null rotations, we have exhibited a lower bound $\nu_{K}$ for $\nu$, but as shown by explicit examples, the precise relationship between $\nu_{K}$ and $K$ is more subtle and a simple uniform expression for all $K$ would be desirable. The intricate dependence of the fraction on the subgroup $K$ does not discard the possibility of finding fractions $\nu$ in this way which have not been encountered before. For the case of branes in motion, where $\nu \leq \frac{1}{4}$, the only fractions which have not appeared so far are $\frac{5}{32}, \frac{3}{16}$ and $\frac{7}{32}$; although all but the latter have occured for static branes.

In this series of papers we have so far focused mostly on M5-branes; yet similar results are also valid for other types of branes both in M-theory as in ten-dimensional superstring theories. In fact, most if not all supersymmetric brane configurations in superstring theory can be related by dualities to supersymmetric brane configurations in $\mathrm{M}$ theory, and hence the classification of M-theory brane configurations would in principle also classify those. At the same time, it is not enough to classify configurations featuring only one type of M-brane: in order to take into account all BPS states it is necessary also to consider configurations consisting of branes of different types, as in the following example.

Let $\varepsilon$ be a spinor of the form

$$
\varepsilon=\left(\begin{array}{l}
\psi_{-} \\
\chi_{+}
\end{array}\right)
$$

From the explicit expression for the $\Gamma$ matrices given in (11), we can see that

$$
\left(e_{0} \wedge e_{9}\right) \cdot \varepsilon=\varepsilon,
$$

which is the algebraic statement corresponding to the fact that the M-wave solution preserves $\frac{1}{2}$ of the supersymmetry [11]. Similarly, we 
have that for spinors of the form

$$
\varepsilon=\left(\begin{array}{l}
\psi_{-} \\
\chi_{-}
\end{array}\right)
$$

the following holds:

$$
\left(e_{0} \wedge e_{9} \wedge e_{\natural}\right) \cdot \varepsilon=\varepsilon,
$$

which now says that an M2-brane stretched along the 09 is supersymmetric. Now let $G=K \ltimes N \subset \mathcal{G}$ and perform a $G$ transformation to each of the above supersymmetric brane solutions: wave (17) and membrane (18). Since $G$ stabilises a spinor $\varepsilon$ of the form (13), the new configurations consisting of the original and the transformed branes is supersymmetric but each preserving $\frac{1}{4}$ of the supersymmetry. Notice then that for spinors of the form (13) we can have configurations containing both $\mathrm{M}$-waves and $\mathrm{M} 2-$ branes in motion which preserve $\frac{1}{4}$ of the supersymmetry. Similarly it is not difficult to construct other configurations involving also M5-branes. We hope to return to a detailed discussion of the general problem in a future publication.

\section{ACKNOWLEDGEMENTS}

It is a pleasure to thank Robert Bryant for his helpful correspondence and for sending us his unpublished notes [3], and Yolanda Lozano for useful discussions at the early stages of this work. Part of this work was done while JMF was visiting the Department of Mathematics of Boston University, and he would like to thank Takashi Kimura for arranging the visit and for hospitality. Finally, BSA and SS are supported by PPARC Postdoctoral Fellowships, JMF by an EPSRC PDRA, and BS by an EPSRC Advanced Fellowship, and we would like to extend our thanks to the relevant research councils for their support.

\section{REFERENCES}

[1] BS Acharya, JM Figueroa-O'Farrill, and B Spence, Planes, branes and automorphisms: I. Static branes, hep-th/9805073.

[2] BS Acharya, JM Figueroa-O'Farrill, and B Spence, Branes at angles and calibrated geometry, JHEP 04 (1998) 012, hep-th/9803260.

[3] RL Bryant, Remarks on spinors in low dimension, Unpublished notes, 1998.

[4] J Dadok and FR Harvey, Calibrations and spinors, Acta Math. 170 (1993), $83-120$.

[5] J Dadok, FR Harvey, and F Morgan, Calibrations on $\mathbb{R}^{8}$, Trans. Am. Math. Soc. 307 (1988), 1-40.

[6] GW Gibbons and G Papadopoulos, Calibrations and intersecting branes, hep-th/9803163.

[7] FR Harvey, Spinors and calibrations, Academic Press, 1990.

[8] HB Lawson and ML Michelsohn, Spin geometry, Princeton University Press, 1989.

[9] N Ohta and PK Townsend, Supersymmetry of M-branes at angles, Phys. Lett. B418 (1998), 77, hep-th/9710129. 
[10] R Penrose and W Rindler, Spinors and space-time, Cambridge University Press, 1984.

[11] PK Townsend, M-theory from its superalgebra, hep-th/9712004.

(BSA,JMF,BS)

$$
\begin{array}{r}
\text { Department of Physics } \\
\text { Queen Mary and Westfield College } \\
\text { Mile End Road } \\
\text { London E1 4NS, UK }
\end{array}
$$

E-mail address: \{r.acharya,j.m.figueroa,b.spence\}@qmw.ac.uk

Theoretical Physics Group

BLACKETT LABORATORY IMPERIAL COLLEGE

Prince Consort RoAd LONDON SW7 2BZ, UK

E-mail address: s.stanciu@ic.ac.uk 